\section{The Seckel syndrome: A case observed in the pediatric department of the University Hospital Center Sourou Sanou (Burkina Faso)}

\author{
Ad Bafa Ibrahim Ouattara, ${ }^{1}$ \\ Makoura Barro, 1 \\ Sahoura Fatimata Nacro, 1 \\ Ibraïma Traoré, ${ }^{2}$ Bintou Sanogo, 1 \\ Jean W. Diallo, ${ }^{3}$ Boubacar Nacro ${ }^{1}$ \\ 1 Pediatrics Department, ${ }^{2}$ Odonto- \\ Stomatology Department, \\ 3Ophtalmology Department, University \\ Hospital Center Sourou Sanou, Bobo- \\ Dioulasso, Burkina Faso
}

\begin{abstract}
Seckel syndrome-1 or "bird-headed dwarfism", Online Mendelian Inheritance in Man number 210600, is a rare genetic disease with an autosomal recessive transmission. We report a female child of 56 months diagnosed with SCKL1 at the Pediatric department of the University Hospital Center Sourou Sanou, Burkina Faso. She showed the typical features including facial dysmorphism, dwarfism, microcephalus and mental retardation. Ophthalmic and dental anomaly and extremities were associated. Without a codified etiological treatment, a psychotherapist support, a genetic counseling, a regular pediatric follow-up, a quarterly odontostomatological and ophthalmological follow-up have been recommended.
\end{abstract}

\section{Introduction}

Seckel syndrome-1 or "bird-headed dwarfism", Online Mendelian Inheritance in Man number 210600 , is a rare genetic disease with autosomal recessive ${ }^{1}$ inheritance caused by homozygous or compound heterozygous mutation in the ATR gene (Gene/locus Mendelian Inheritance in Man number 601215) on chromosome $3 \mathrm{q} 23 .{ }^{2}$ It is a morphological entity defined by four criteria combining intra-uterine growth retardation (IUGR) exceeding two standard deviations, microcephaly often correlated to a craniosynostosis, facial dysmorphism and mental retardation of variable degree. ${ }^{3,4}$

SCKL1 has been reported from different geographic areas worldwide, including Africa, ${ }^{5}$ with a prevalence of $1 / 10.000 .{ }^{6} \mathrm{We}$ report the observation of Seckel syndrome in a child at the University Hospital Center
Sourou Sanou (UHCSS) in BoboDioulasso.

\section{Case Report}

D.P, a female child of 56 months, was referred by the Saint Leopold clinic of Bobo-Dioulasso to UHCSS in pediatric consultation on March 1 ${ }^{\text {st }}, 2017$ for growth and mental retardation. She was born vaginally at the 43 week with an IUGR (birth weight $2120 \mathrm{~g}$ ), from a consanguineous marriage (Figure 1). A retardation of psychomotor development was progressively documented with head control since 7 months, sitting since 8 months, standing and walking position acquired at 2 years, and markedly delayed language landmarks. On admission, the child showed a severe growth retardation: the weight at $11,80 \mathrm{~kg}$ (Weight for Age percentile $=0.1 \%$; Weight for Height percentile $=0.8 \%$ ), the height at $97,6 \mathrm{~cm}$ (Height for Age percentile $=2.0 \%$ ) and the head circumference at $39 \mathrm{~cm}$ (Head circumference for Age percentile $=0.0 \%) .{ }^{7}$ Her face was dysmorphic with a prominent beaked nose, facial asymmetry, low-set ears and a micrognathia; curly and brittle red hair; campodactyly and clinodactyly of the $5^{\text {th }}$ finger (Figure 2). Ocular evaluation showed bilateral convergent strabism and retinal vascular tortuosity, with normal intra ocular pressure. Mental retardation was characterized by a delay in language and understanding, posture of the head, sitting, walking and standing. She also had emotional fragility with high dependence for activities including coloring, speech, performing games. Her IQ was estimated between 35-49. Bone age was 36 months. The child was prospectively followed for supportive care. At the age of 6 years, she is clinically stable, and her main limitation is due to the severe dwarfism and facial dysmorphism, which heavily limit her social activity. Furthermore, her eyesight may be progressively limited by retinal vasculopathy.

\section{Discussion}

In a review of literature, Kalay et al. reported a case of Seckel syndrome in South Africa; ${ }^{6}$ to the best of our knowledge, this is the first case diagnosed in Burkina Faso. The failure to thrive was the cause of consultation in our patient, diagnosed at the age of 4 years and 8 months, as reported by Thapa et al ${ }^{8}$. The diagnosis has been reported at any age, even antenatal through medical imagery viewing 1,9 (echography in $3 \mathrm{D}$, IRM).
Correspondence: Ad Bafa Ibrahim Ouattara, Department of Pediatrics, , University Hospital Center Sourou Sanou, Bobo-Dioulasso, 01 BP 676, Burkina Faso.

Tel.: +22671295102, Fax: +2220972693

E-mail: adbafa1983@hotmail.com

Key words: Seckel, bird-headed dwarfism-child.

Acknowledgments: The authors are grateful to the caregivers for their participation in this study.

Contributions: ABIO carried out the clinical evaluation and was the major contributor in writing the manuscript, MB carried out the clinical evaluation, SFN carried out the clinical evaluation, IT performed the odonto-stomatological examination, BS carried out the clinical evaluation, JWD performed the ocular examination, $\mathrm{BN}$ carried out the clinical examination

Conflict of interest: The authors declare no potential conflict of interest

Availability of data and materials: Data available upon reasonable request to the authors.

Ethics approval and consent to participate: This study was conducted in accordance with all relevant guidelines and procedures.

Informed consent: Informed consent has been obtained form the family.

Received for publication: 3 July 2019.

Revision received: 5 December 2019.

Accepted for publication: 10 December 2019.

This work is licensed under a Creative Commons Attribution NonCommercial 4.0 License (CC BY-NC 4.0).

(C) Copyright: the Author(s), 2020

Licensee PAGEPress, Italy

Pediatric Reports 2020; 12:8231

doi:10.4081/pr.2020.8231

The observation of parental consanguinity is in keeping with an autosomal recessive disorder. 1,2

The differential diagnosis is mainly with osteodysplastic dwarfism with type II microcephaly. 10

In our medical and social setting with limited resources, the diagnosis of rare conditions may be based on clinical observation and recognition of the typical phenotype. Unfortunately, we do not have access to molecular studies which might confirm mutation in the associated gene. Faivre et al. ${ }^{11}$ confirmed the heterogeneity of Seckel syndrome by excluding the previously mapped loci on chromosomes 3 and 18 in 5 consanguineous and 1 multiplex nonconsanguineous Seckel syndrome families. 

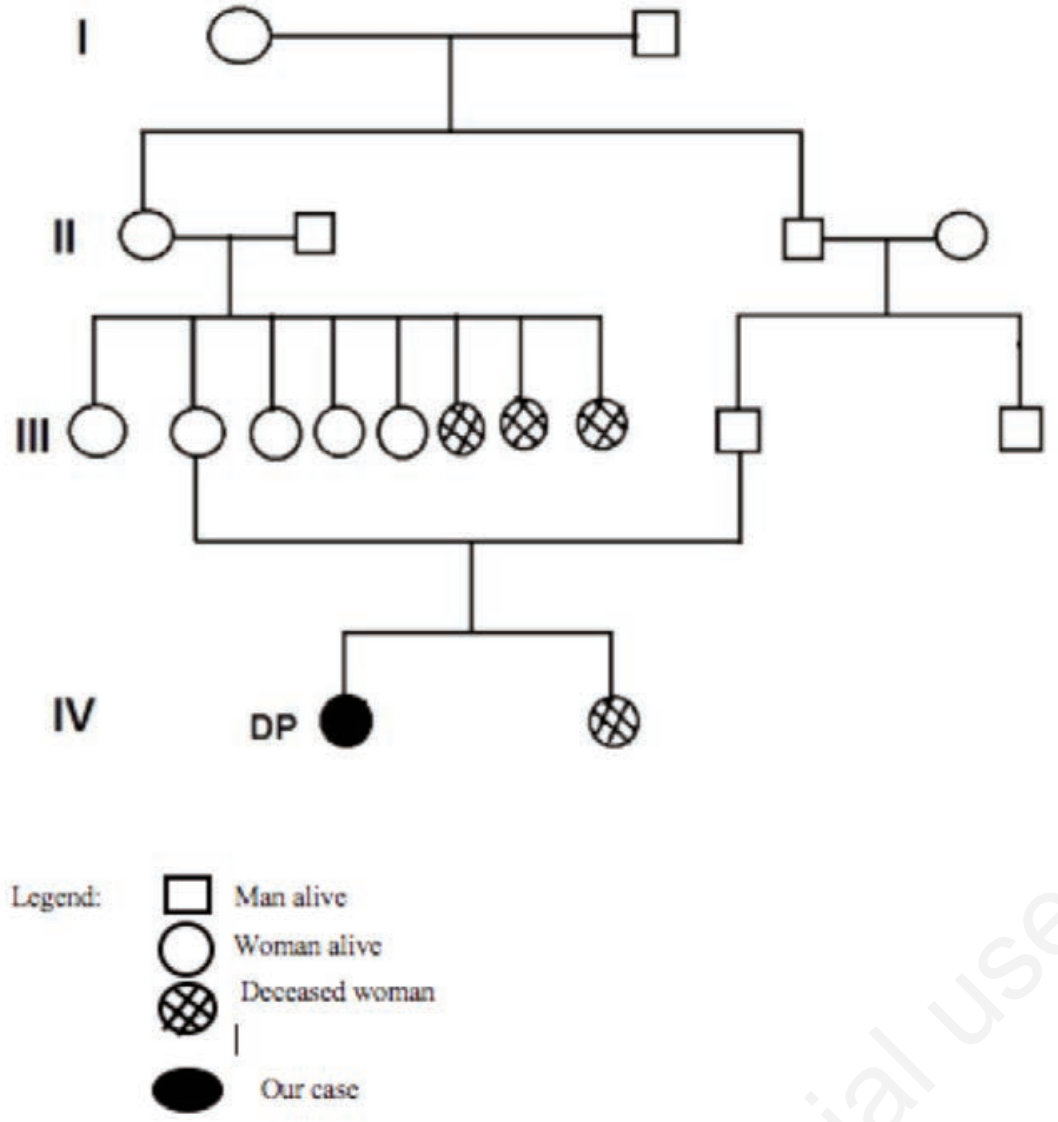

Figure 1. Family tree.
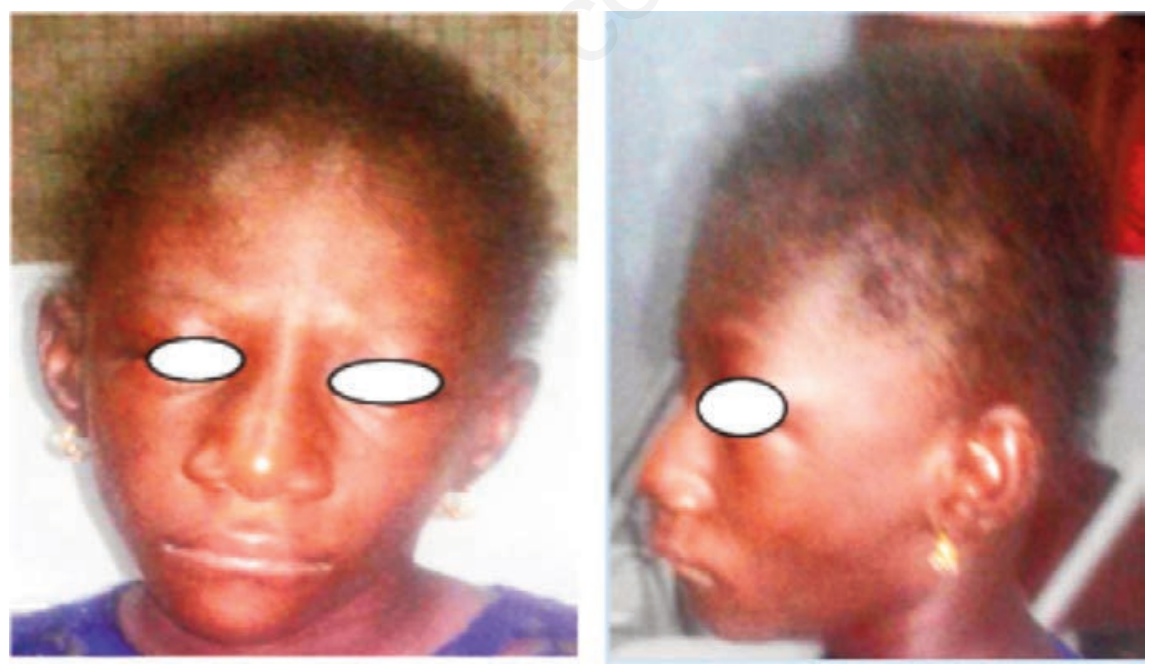

Figure 2. Characteristic facial dysmorphism with prominent beak-shaped nose, micrognathia, low-set ears.

\section{Conclusions}

This observation made it possible to highlight the limits in diagnosis confirmation namely in the carrying out of molecular biology.

\section{References}

1. Vascone C, Di Meglio F, Di Meglio L, et al. Antenatal diagnosis of Seckel Syndrome: a rare case report. J Prenat Med 2014;8:70-2.

2. Orphanet: Recherche de maladies. Available from: https://www.orphanet/consor/cgibin/Disease_Search.php Ing=FR\&data $\mathrm{id}=954$.

3. Parent P, Moulin S, Munck MR, et al. [Bird headed dwarfism in Seckel syndrome. Nosologic difficulties]. Arch Pediatr 1996;3:55-62. [Article in French]

4. Shanske A, Caride DG, MenassePalmer L et al. Central nervous system anomalies in Seckel syndrome: report of a new family and review of the literature. Am J Med Genet 1997;70:155-8.

5. Akkurt MO, Pakay K, Akkurt I, et al. Prenatal diagnosis of Seckel syndrome at 21 weeks' gestation and review of the literature. J Matern Fetal Neonatal Med 2017;1-4.

6. Kalay E, Yigit G, Aslan Y et al. CEP152 is a genome maintenance protein disrupted in Seckel syndrome. Nat Genet 2011;43:23-6.

7. WHO. The WHO child growth standard. Available from: http://www. who.int/childgrowth/standards/en/

8. Thapa R, Mallick D, Biswas B, Ghosh A. Open and closed lip schizencephaly in Seckel syndrome: a case report. J Child Neurol 2010;25:494-6.

9. Takikawa KM, Kikuchi A, Yokoyama A et al. Perinatal findings of Seckel syndrome: a case report of a fetus showing primordial dwarfism and severe microcephaly. Fetal Diagn Ther 2008;24: 405-8.

10. Majewski F, Ranke M, Schinzel A. Studies of microcephalic primordial dwarfism type II: the osteodysplasic type II of primordial dwarfism. Am J Med Genet 1982;12:23-35.

11. Faivre L, Le Merrer M, Lyonnet S, et al. Clinical and genetic heterogeneity of Seckel syndrome. Am J Med Genet 2002;112:379-83. 